\title{
Evaluation of Antioxidant Activity of Some Mesoionic Pyrimidinium Betaines by Three Different Methods
}

\author{
F. Malki, A. Touati, S. Moulay, and M. Baltas
}

\begin{abstract}
A series of mesoionic pyrimidinium betaines were synthesized and tested in vitro for their antioxidant activity using three tests: 2,2-diphenyl-1-picrylhydrazil (DPPH) free radical scavenging, Ferric Reducing Antioxidant Power (FRAP) and bleaching of $\beta$-carotene.

The results indicated that pyrimidinium betaines possess antioxidant properties with three methods. In the DPPH assay, the monocyclic pyrimidinium betaine showed the highest activity with an $\mathrm{IC}_{50}$ value of $300 \mu \mathrm{g} / \mathrm{mL}$. The pyrimidinium betaines also revealed a reducing power lower than that of the control antioxidants, and the monocyclic pyrimidinium betaine was the most active one. In the $\beta$-carotene bleaching test, the oxidation of linoleic acid was inhibited by all the betaines. The bicyclic one showed the greatest inhibitory activity of the oxidation of linoleic acid with a relative antioxidant activity (RAA) of $90 \%$, an activity higher than that of the reference antioxidant $\alpha$-tocopherol $(41 \%)$. It was found that the antioxidant efficacy increased with increasing concentration of betaine and depended on the nature of the substituent on the pyrimidine ring.
\end{abstract}

Index Terms-Antioxidant ativity, $\beta$-carotene bleaching, dpph assay, frap assay, pyrimidinium betaine.

\section{INTRODUCTION}

Lipid peroxidation is the leading cause of fatty foods rancidity [1]. Food may contain microorganisms that can lead to the deterioration and, eventually, a food-borne illness. Therefore, preservation of food quality consists in the control of lipid oxidation and of growth of spoilage and pathogenic microorganisms.

There is growing interest in antioxidants, particularly those designed to prevent the suspected deleterious effects of free radicals in the human body; the free radicals are able to degrade fatty matter and other food components. It was reported that free radicals that are generated in bioorganic redox process can cause oxidative damage in various body parts, and may be implicated in process resulting in the formation of mutations [2].

Manuscript received May 15, 2016; revised August 12, 2016. This work was supported by research funding from Ecole Normale Supérieure.

F. Malki and A. Touati are with the Laboratoire de recherche sur les produits bioactifs et valorisation de la biomasse, Ecole Normale Supérieure, BP 92,16000, Kouba, Algiers, Algéria (e-mail: malki_fatiha76@yahoo.fr, a_touati2000@yahoo.fr).

S. Moulay is with the Laboratoire de Chimie-Physique Moléculaire et Macromoléculaire, Département de Chimie Industrielle, Faculté de TEchnologie, Université Saâd Dahlab de Blida, Blida, Algeria (e-mail: polymchemlab@hotmail.com).

Michel Baltas is with the Laboratoire de Synthèse et Physicochimie de Molècules d'Intèrêt Biologique, UMR 5068, Université Paul Sabatier, 118, route de Narbonne - 31062 Toulouse Cedex 9, France (e-mail: baltas@chimie.ups-tlse.fr).
In recent years, several researchers have been interested in searching that can potentially act as free radical scavengers, and a number of antioxidant molecules have been incorporated in foods to protect their shelf-life [3], [4].

In parallel, a number of methods have been developed to evaluate the antioxidant capacity of natural and synthetic compounds [4]-[7].

Betaine derivatives have a variety of uses in medicine, pharmacy, biology and other scientific fields [8]. Today, the chemistry of betaines has become a subject of particular interest due to their applications in biological research, especially with regard to their metabolic roles in the living organism [9].

Pyrimidine compounds exhibit diverse biological activities, since the pyrimidine ring is a fundamental nucleus in DNA and RNA [10].

In line with these properties, the aim of the present work was to evaluate the antioxidant activities of synthesizing betaines having the pyrimidine ring in their structures, and compare them to those of standard antioxidants such as butylated hydroxyanisole (BHA), butylated hydroxy toluene (BHT), ascorbic acid (vitamin C) and $\alpha$-tocopherol (vitamin E). To this end, three tests were employed: 2, 2-diphenyl-1-picrylhydrazyl (DPPH) radical scavenging activity, ferric ions reducing antioxidant power (FRAP) and $\beta$-carotene/linoleic acid assays.

\section{MATERIALS AND METHODS}

\section{A. Chemicals}

Chemicals used were of analytical grade and purchased from either Sigma-Aldrich, Merck, Prolabo or Biochem.

\section{B. Synthesis}

Three pyrimidinium betaines: two bicyclics 1, 2, one monocyclic 3, and one with fatty chain alkyl 4, were prepared from amine derivatives and malonic esters by the reported procedures [11]-[13]. Their chemical structures are represented in Fig. 1

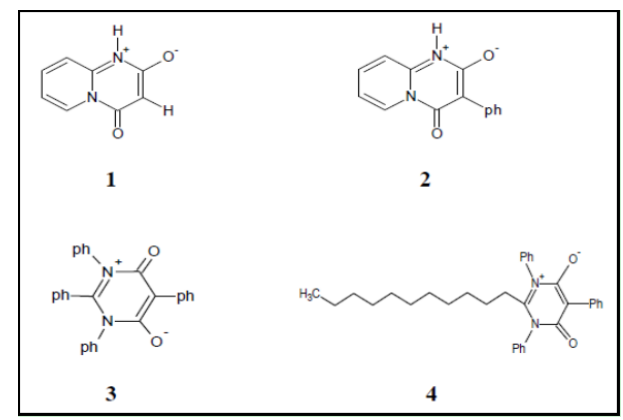

Fig. 1. Chemical structures of synthesized pyrimidinium betaines 1-4. 


\section{Antioxidant Assay}

Antioxidants are reducing compounds able to scavenge free radicals and thus help the body to prevent oxidation. Although there are no standard methods for evaluating the antioxidant potential of food and biological systems, it is recommended to evaluate the antioxidant activity by different methods [14].

In this study we have applied three tests to evaluate the potential antioxidant of the synthesized pyrimidinium betaines 1-4. Antioxidant activities of standard antioxidants (BHT, BHA, ascorbic acid (vitamin C) and $\alpha$-tocopherol (vitamin E) used as references were determined in parallel experiments.

\section{1) DPPH radical scavenging assay}

The capacities of pyrimidinium betaines donation of hydrogen atoms or electrons are measured from the discoloration of the purple methanolic solution of free radical 2, 2 diphenyl-1-picrylhydrazyl (DPPH) according to the method of Blois [4] with some modifications. The principle of this method is based on measuring the scavenging power of the radical DPPH in methanolic solution.

\section{a) Experimental protocol}

A solution of DPPH $(0.004 \%)$ in methanol was prepared and $1 \mathrm{~mL}$ of this solution was mixed with $1 \mathrm{~mL}$ of varying concentrations of betaines solution in ethanol. The reaction mixture was swirled thoroughly and left in the dark at room temperature for $3 \mathrm{~h}$. The absorbance compared to a negative control containing only DPPH solution was measured spectrophotometrically at $517 \mathrm{~nm}$. The radical scavenging activity was calculated using the following formula:

DPPH radical scavenging activity $\left.(\%)=\left[\left(\mathrm{A}_{\mathrm{C}}-\mathrm{A}_{\mathrm{S}}\right)\right] /(\mathrm{AC})\right] \times 100$

where $A_{C}$ is the absorbance of the control reaction (DPPH solution without the compound to be tested), $A_{S}$ is the absorbance of sample.

The concentration of betaine providing 50\% inhibition $\left(\mathrm{IC}_{50}\right)$ was calculated from the graph of the plot of inhibition percentage against betaine concentration [14], [15].

\section{2) Reducing power assay}

The reducing power of a compound is related to its transfer capacity of electrons and can serve as a significant indicator of its potential antioxidant activity. The reducing power is to measure the reductive ability of an antioxidant, and it is evaluated by the transformation of Fe (III) to Fe (II) in the presence of the extract of the sample [15]. The ability to reduce $\mathrm{Fe}$ (III) can be attributed to the hydrogen donation capacity from phenolic compounds as described by Shimada et al. [16]; this donation is related to the presence of reducing agent [17].

In this assay, the yellow color of the test solution turns green and blue according to the reducing power of the test sample. The capacity of the oxidant to reduce the ferric ferricyanide complex to the ferrous one was determined based on absorbance at $700 \mathrm{~nm}$ after incubation. The greatest absorbance at $700 \mathrm{~nm}$ indicates a greater reducing power [18], [19].

In our study, the reducing power of the synthesized betaines was determined by the method of Oyaizu [20]. Antioxidants references (BHT, BHA, $\alpha$-tocopherol (vitamin
E) and ascorbic acid (vitamin C)) were used as the positive controls.

\section{a) Experimental protocol}

Different concentrations of betaines in $1 \mathrm{~mL}$ of ethanol were mixed with $2.5 \mathrm{~mL}$ of phosphate buffer $(0.2 \mathrm{M}$, pH 6.6) and $2.5 \mathrm{~mL}$ of potassium ferricyanide $\left[\mathrm{K}_{3} \mathrm{Fe}(\mathrm{CN})_{6}\right](1 \%)$, and then the mixture was incubated at $50{ }^{\circ} \mathrm{C}$ for $20 \mathrm{~min}$. Afterwards, $2.5 \mathrm{~mL}$ of trichloroacetic acid (10\%) was added to the mixture, which was then centrifuged at $1000 \mathrm{rpm}$ for 10 min. Finally, $2.5 \mathrm{~mL}$ of the upper layer solution was mixed with $2.5 \mathrm{~mL}$ of distilled water and $0.5 \mathrm{~mL}$ of $\mathrm{FeCl}_{3}(0.1 \%)$, and the absorbance was measured at $\lambda_{\max }=700 \mathrm{~nm}$. Increased absorbance of the reaction mixture indicates increased reducing power.

\section{3) $\beta$-Carotene bleaching assay}

In this test, the antioxidant power of a tested compound is determined by measuring the inhibition of oxidative degradation of $\beta$-carotene by the oxidation of linoleic acid [21], [22]. The addition of an antioxidant allows a delay of the discoloration kinetics of $\beta$-carotene.

$\beta$-Carotene bleaching assay was conducted by using the method suggested by Tepe [17] with some modifications.

\section{a) Experimental protocol}

A solution of $\beta$-carotene was prepared by dissolving 0.5 $\mathrm{mg}$ of $\beta$-carotene in $1 \mathrm{~mL}$ of chloroform. $25 \mu \mathrm{L}$ of linoleic acid and $200 \mathrm{mg}$ of Tween 80 were added. Chloroform was completely evaporated using a vacuum evaporator. Then, 100 $\mathrm{mL}$ of distilled water saturated with oxygen were added with a vigorous shaking. $2.5 \mathrm{~mL}$ of this reaction mixture were dispersed in a test tube and $350 \mu \mathrm{L}$ of the sample prepared in DMSO at $2 \mathrm{mg} / \mathrm{mL}$ concentrations were added; the ensued emulsion system was incubated for $48 \mathrm{~h}$ at room temperature. The absorbance of the mixture was measured at $\lambda_{\max }=490$ $\mathrm{nm}$ at regular time intervals for $48 \mathrm{~h}$ [23]. The same procedure was repeated for the positive controls, BHT and $\alpha$-tocopherol (vitamin E), and for a blank. The relative antioxidant activity (RAA) was calculated according to the following equation:

$$
\mathrm{RAA} \%=\left(\mathrm{A}_{\mathrm{S} 48 \mathrm{~h}} / \mathrm{A}_{\mathrm{C} 48 \mathrm{~h}}\right) \times 100
$$

where $A_{S} 48 \mathrm{~h}$ is the absorbance of the sample after 48 hours and $\mathrm{A}_{\mathrm{C}}$ 48h the absorbance of $\mathrm{BHT}$ used as the positive control.

\section{RESULTS AND DISCUSSION}

Pyrimidinium betaines $\mathbf{1 - 4}$ were synthesized and characterized as described earlier [10], [11]. Their antioxidant behavior was evaluated in vitro by three different tests: DPPH test, measurement of reducing power, and $\beta$-carotene bleaching technique.

\section{A. DPPH Radical Scavenging Activity}

DPPH radical scavenging is a sensitive antioxidant assay and is independent of the polarity of the substrate [24]. This test is widely used to evaluate antioxidant activities in a relatively short time compared to other methods. The stability of this free radical results from the important delocalization of unpaired electron on the whole molecule. In this assay, 
diphenyl picrylhydrazyl (purple color) reacts with antioxidant and is reduced into diphenyl picrylhydrazine (yellow). The intensity of the color is inversely proportional to the capacity of the antioxidant present in the medium to give hydrogen or electrons [25]. The DPPH test is performed for each betaine at several concentrations.

The antiradical activity was evaluated spectrophotometrically by following the reduction of the radical at $517 \mathrm{~nm}$ for $3 \mathrm{~h}$. For comparative purposes, standard antioxidants were used. The results of percentage inhibition of the DPPH radical by standard antioxidants and betaines are represented in Fig. 2.

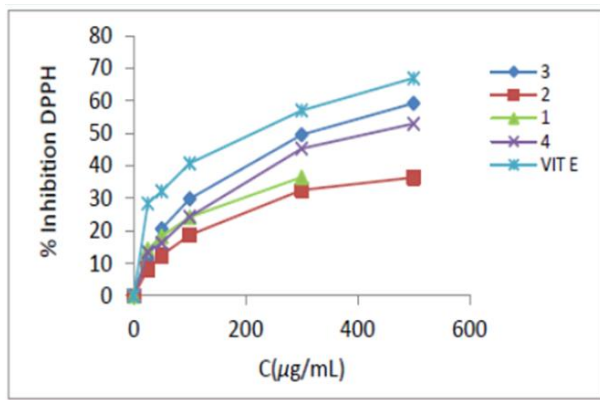

Fig. 2. Radical scavenging activity of DPPH against pyrimidinium betaines 1-4.

As can be seen, the betaines present a scavenging capacity of free radicals. This activity can be attributed to the conjugate systems with nitrogen atoms in these molecules, which are known to stabilize free radicals [26]. It is also noted that the efficiency of the antioxidant increased with the concentration of the tested betaine.

The $\mathrm{IC}_{50}$ values, which are concentrations of antioxidants required to reduce $50 \%$ the DPPH concentration, are given in Table I and illustrated in Fig. 3.

TABLE I: $\mathrm{IC}_{50}$ VALUES ( $\mu \mathrm{G} / \mathrm{ML}$ ) OF THE BETAINES AND THE CONTROL ANTIOXIDANTS IN THE DPPH ASSAY

\begin{tabular}{lc}
\multicolumn{2}{c}{ ANTIOXIDANTS IN THE DPPH ASSAY } \\
\hline Antioxidant & $\mathrm{IC}_{50}(\mu \mathrm{g} / \mathrm{mL})$ \\
\hline BHA & 3 \\
BHT & 3.85 \\
Vitamin E & 210 \\
1 & - \\
2 & - \\
3 & 300 \\
4 & 420 \\
\hline
\end{tabular}

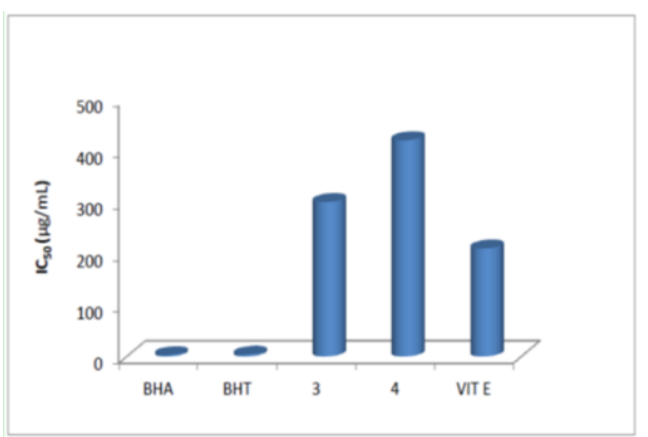

Fig. 3. $\mathrm{IC}_{50}$ values $(\mu \mathrm{g} / \mathrm{mL})$ of betaines and reference antioxidants in the DPPH assay.

According to $\mathrm{IC}_{50}$ results, the monocyclic betaine $\mathbf{3}$ and the betaine with a fatty chain $\mathbf{4}$ are the most reactive, with an $\mathrm{IC}_{50}$ of 300 and $420 \mu \mathrm{g} / \mathrm{mL}$, respectively. These values are close to that of the reference antioxidant vitamin $\mathrm{E}\left(\mathrm{IC}_{50}=210\right.$ $\mu \mathrm{g} / \mathrm{mL}$ ) and considerably lower than those of BHT and BHA (3.85 and $3 \mu \mathrm{g} / \mathrm{mL}$, respectively). These results are in agreement with our previous ones [8]. The antioxidant activity of these two molecules can be attributed to the conjugated systems involving nitrogen atoms which are more extensive in these molecules in comparison with the bicyclics $\mathbf{1}$ and $\mathbf{2}$. In $\mathbf{3}$ and $\mathbf{4}$, the radical formed is strongly stabilized by resonance through phenyl and carbonyl groups.

Regarding the bicyclic betaines $\mathbf{1}$ and $\mathbf{2}$, no significant decrease in the absorbance at $517 \mathrm{~nm}$ was observed and their $\mathrm{IC}_{50}$ values were not determined as higher concentrations were required; mono- and bicyclic betaines were sparingly soluble in ethanol at high concentrations [27].

The results of this study reveal that the low reactivity of the pyrimidinium betaines in comparison with that of standard antioxidants (BHT and BHA), could be due to the effects of steric hindrance and the nature of the substituents affixed on the hydrophilic head of betaine .

\section{B. Reducing Power Assay}

The reducing powers of standard antioxidants and of synthesized betaines at $700 \mathrm{~nm}$ are presented in Fig. 4.

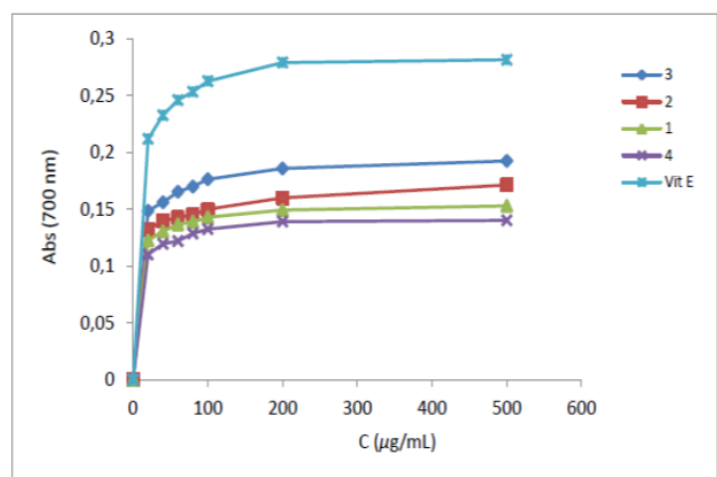

Fig. 4. Reducing powers of the pyrimidinium betaines 1-4 and of the positive control (vitamin E) at $700 \mathrm{~nm}$.

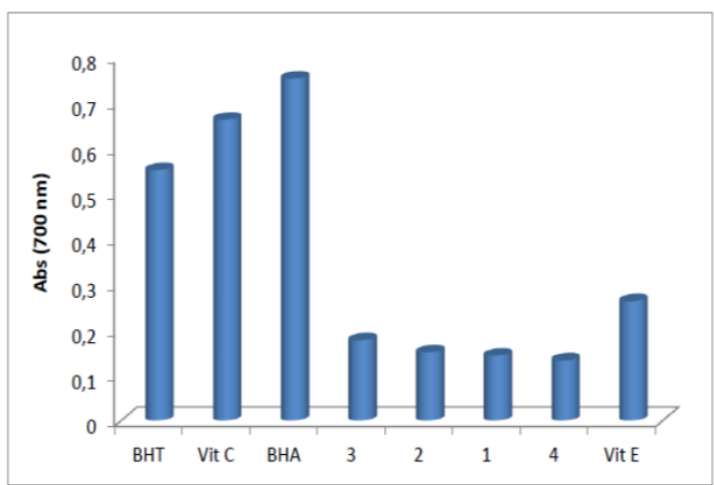

Fig. 5. Reducing power of betaines and reference antioxidants $(C=100$ $\mu \mathrm{g} / \mathrm{mL})$.

The reducing power of the tested betaines increased with their increasing concentrations and reached plateaus beyond $200 \mu \mathrm{g} / \mathrm{mL}$. It can be noticed that the reducing powers of these compounds were close to that of vitamin $\mathrm{E}$.

By comparing the obtained results, we note that the betaine monocyclic 3 is the most active followed by bicyclic betaines 2 and 1, whereas a weaker reducing power was observed in the long chain betaine 4 . The steric effect may influence the reducing power. Indeed, the low reactivity of $\mathbf{4}$ may be due to the presence of the fatty alkyl chain, being long, thus 
providing a steric hindrance. Fig. 5 depicts the reducing power of betaines and reference antioxidants at $\mathrm{C}=100 \mu \mathrm{g} /$ $\mathrm{mL}$.

Again, it can be remarked that the antioxidant activity of the tested betaines was close to that of vitamin E, but it was lower than that of the remaining reference antioxidants (BHT, BHA and vitamin C).

\section{C. $\beta$-Carotene Bleaching Assay}

According to several authors, the test of inhibition of the oxidation of linoleic acid coupled with $\beta$-carotene, appeared very useful as a model of lipid peroxidation in biological membranes [28].

The $\beta$-carotene / linoleic acid test determines the inhibition rate of the oxidation of linoleic acid to confirm anti-lipoperoxidation effects of sample. The bleaching kinetics of $\beta$-carotene in the presence of the synthesized betaines and reference antioxidants (BHT and Vitamin E) is shown in Fig. 6.

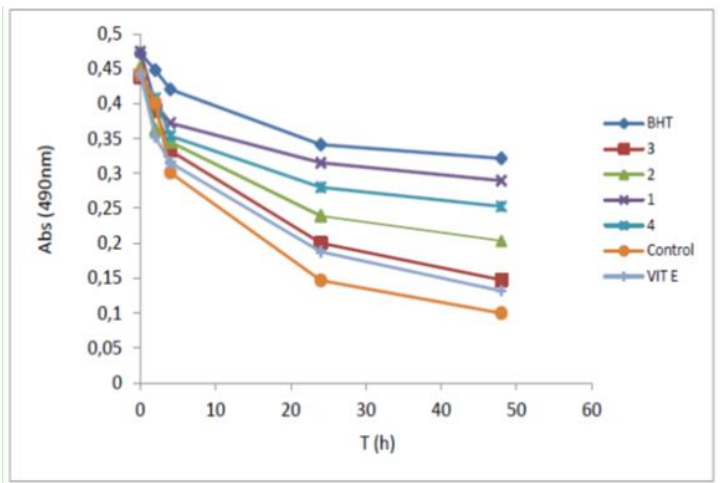

Fig. 6. Bleaching kinetics of $\beta$-carotene at $490 \mathrm{~nm}$ in the presence of betaines and reference antioxidants $\mathrm{BHT}$ and vitamin $\mathrm{E}$.

The results in Fg. 6 show that all the betaines inhibited efficiently the oxidation of linoleic acid/ $\beta$-carotene system when compared to the negative control, and their antioxidant activities (RAA $>45 \%$ ) were greater than that of vitamin $\mathrm{E}$ $($ RAA $=41.03 \%$.). The antioxidant activity (RAA) of betaines and vitamin E relative to that of the BHT is shown in Fig. 7.

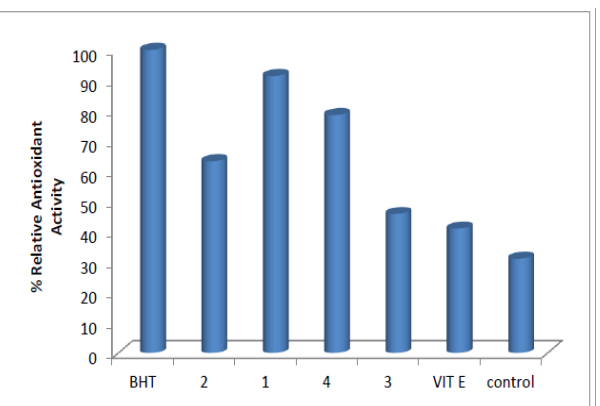

Fig. 7. Relative antioxidant activity (RAA) of various betaines and reference antioxidants $\mathrm{BHT}$ and vitamin $\mathrm{E}$ in $\beta$-carotene/linoleic acid system.

According to the obtained results, it can be noted that the bicyclic betaine 1 shows the greatest oxidation inhibitory activity of linoleic acid, with an RAA of $90 \%$ with respect to that of BHT. On the other hand, the long chain betaine 4 shows an antioxidant activity RAA as high as $78.52 \%$, whereas that of the bicyclic betaine 2 was $63.26 \%$, and the least active is the monocyclic betaine $\mathbf{3}$ with an RAA of $45.88 \%$. According to this method, betaine 3 is almost twice less active than bicyclic betaine $\mathbf{1}$.

Steric effects can play a fundamental role in the discoloration test of $\beta$-carotene. Indeed, the low reactivity of monocyclic betaine $\mathbf{3}$ may be due to the presence of four phenyl groups, thus giving rise to a steric hindrance. On the contrary, the greater reactivity of bicyclic betaine $\mathbf{1}$ may be associated with the small size of the molecule compared to those of the remaining betaines.

On the other hand, there is a marked improvement in antioxidant activity on going from the monocyclic betaine $\mathbf{3}$ to fatty chain betaine 4 . It appears that increasing the lipophilicity would have led to an increase in antioxidant activity. It is therefore understood that chemical nature of the surroundings of mesoionic site greatly influence the antioxidant power of betaine .

According to Frankel and Meyer [14], the bleaching test of $\beta$-carotene is similar to a lipid emulsion system in water. These authors suggested that the apolar antioxidants are the most important because they are concentrated in lipid-water interface, preventing the formation of lipid radicals and the oxidation of $\beta$-carotene. Comparing the chemical structures of monocyclic betaine 3 and betaine $\mathbf{4}$, with a long alkyl chain, it can be concluded that the former one is more polar and less active than the latter one, a fact that endorses further our results.

\section{CONCLUSION}

In this work we evaluated the antioxidant activity of four types of pyrimidinium betaines through three methods: 2 , 2-diphenyl-1-picryl-hydrazil (DPPH) free radical scavenging, Ferric Reducing Antioxidant Power (FRAP) and bleaching of $\beta$-carotene.

The results showed that the pyrimidine betaines exhibit considerable antioxidant activities compared to those of the reference antioxidants.

In the DPPH test, the monocyclic and long chain betaine showed an antiradical potential close to that of vitamin E, whereas lower antioxidant power was observed for bicyclic betaines.

The ability of these compounds to inhibit lipid peroxidation, assessed by bleaching technique of $\beta$-carotene, indicates that the oxidation of linoleic acid can be effectively inhibited by the four betaines. These betaines have also shown a reducing power lower than those of the reference compounds. It is noted that antioxidant efficacy increases with the concentration of betaines and is directly related to the nature of the substituents on the pyrimidine ring.

Comparing the results obtained by the three methods, we find that the antioxidant activity differs according to the test used. The monocyclic betaine $\mathbf{3}$ is the most active in the test of scavenging free radical DPPH and possesses the best reducing power compared to other betaines. The activity of bicyclic betaine 1 is better in the bleaching test of $\beta$-carotene and low in the other two tests. On the other hand, the betaine with fatty chain 4 showed a considerable activity in the DPPH test and the bleaching of $\beta$-carotene.

On the basis of the results of this study, it can be concluded that the synthesized pyrimidinium betaines have powerful antioxidant activity in different in vitro assays when compared to the standard antioxidant compounds. These 
results suggested that these molecules might serve as interesting lead compounds for the development of new antioxidant that can be important in disease prevention and health preservation, and possible food supplements, or in pharmaceutical applications.

\section{REFERENCES}

[1] M. Boudkhili, H. Greche, S. Bouhdid, F. Zerargui, and L. Aarab, "In vitro antioxidant and antibacterial properties of some Moroccan Medicinal Plants," Intern J Pharm Tech Res, vol. 4, pp. 637-642, April-June 2012.

[2] P. Arulpriya, P. Lalitha, and S.Hemalatha, "In vitro antioxidant testing of the extracts of Samanea saman (Jacq.) Merr.," Der Chemica Sinica, vol. 1, pp. 73-79, 2010.

[3] J. Lebeau, C. Furman, J. L. Bernier, P. Duriez, E. Teissier, and N. Cotelle, "Antioxidant properties of ditert-butylhydroxylated flavonoids," Free Rad. Biol. Med., vol. 29, pp. 900-912, November 2000.

[4] M. S. Blois, "Antioxidant determinations by the use of stable free radical," Nature, vol. 181, pp. 1199-1200, 1958.

[5] G J. Marco. "A rapid determination method for evaluation of antioxidants," J. Am. Oil Chem. Soc., vol. 45, pp. 594-598, 1968.

[6] I.F.F. Benzie and J.J .Strain, "The ferric reducing ability of plasma (FRAP) as a measure of antioxidant power: The FRAP assay," Anal. Biochem, vol. 239 pp. 70-76, July 1996.

[7] F. Malki, A. Touati, and S. Moulay, "Antioxidant activity of two mesomeric heterocyclic betaines containing a pyrimidine moiety," Pertanika J. Trop. Agric. Sci. 36, pp. 393-402, April 2013.

[8] M. A. Gonzalez, J. C. Royero, A. Mesa, and L. B. Galvis, "Synthesis and biological evaluation of pyridine betaine A and B," Natl. Prod. Res., vol. 23, pp. 1485-1491, November 2009.

[9] T. A. Naik and K. H.Chikhalia, "Studies on synthesis of pyrimidine derivatives and their pharmacological evaluation," e-J Chem, vol. 4, pp. 60-66, January 2007.

[10] F. Malki, A. Touati, S. Rahal, and S. Moulay, "Total synthesis of monocyclic pyrimidinium betaines with fatty alkyl chain," Asian $J$. Chem., vol. 23, pp. 961-967, 2011.

[11] F. Malki, A. Touati, and S. Moulay, "Extraction and Recrystallization of Mesoionic Pyrimidinium Betaines," IJCEA, vol. 5, pp. 151-154 April 2014.

[12] A. E. Tschitschibabin, "Formation of bicyclic derivatives of $\alpha$-aminopyridine,” Ber. Dtsch .chem. Ges., vol. 57, pp. 1168-1172, 1924.

[13] P. Dvortsak, G. Resofszki, M. Huhn, L. Zalantai, and A. I. Kiss, "Reactions of pentachlorophenyl esters of malonic acid derivatives II. Preparation and investigation of pyrimidine betaines," Tetrahedron, vol. 32, pp. 2117-2120, 1976.

[14] E. N. Frankel and A. S. Meyer, "The problems of using one-dimensional methods to evaluate multifunctional food and biological antioxidants," J Sci Food Agric, vol. 80, pp. 1925-1940, 2000.

[15] I. Gülçin, M. Oktay, E. Kıreçci, and Ö.I Küfreviolu, "Screening of antioxidant and antimicrobial activities of anise (Pimpella anisum L.) seed extracts," Food. Chem., vol. 83, pp. 371-382, 2003.

[16] K. Shimada, K. Fujikawa, K. Yahara, and T. Nakamura, "Antioxidative properties of xanthan on the autoxidation of soybean oil in cyclodextrin emulsion," J. Agric. Food Chem., vol. 40, pp. 945-948, 1992.
[17] P. D. Duh, “Antioxidant activity of Budrock (Arctium laooa Linn): Its scavenging effect on free radical and active oxygen," J. Am. Oil Chem. Soc., vol. 75, pp. 455-461, 1998.

[18] M. Elmastaş, I. Gülçin, Ö. Işildak, Ö. I. Küfrevioğlu, K. İbaoğlu, and H. Y. Aboul-Enein, "Radical scavenging activity and antioxidant capacity of bay leaf extracts," J. Iran. Chem. Soc., vol. 3, pp. 258-266, 2006.

[19] I. Gülçin, M. Elmastat, and H. Y. Aboul-Enein, "Determination of Antioxidant and radical scavenging activity of basil (Ocimum basilicum L. Family Lamiaceae) assayed by different methodologies," Phytother. Res., vol. 21, pp. 354-361, 2007.

[20] M. Oyaizu, "Studies on products of browning reaction prepared from glucoseamine," Jpn J. Nutr., vol. 44, pp. 307-314, 1986.

[21] G. L. Marco, "A rapid method for evaluation of antioxidants," J. Am. Oil Chem. Soc., vol. 45, pp. 594-598, 1968.

[22] H. E. Miller, "Simplified method for the evaluation of antioxidants," Je Am Oil Chem Soc, vol. 48, pp. 91-97, 1971.

[23] B. Tepe, M. Sokmen, H. A. Akpulat, and A. Sokmen, "Screening of the antioxidant potentials of six Salvia species from Turkey," Food Chem., vol. 95, pp. 200-204, 2006.

[24] T. Yamaguchi, H. T. T. Matoba, and J. Terao, "HPLC Method for Evaluation of the Free Radical-scavenging Activity of Foods by Using 1, 1-Diphenyl-2-picrylhydrazyl. Bioscience," Biotechnol Biochem, vol. 62, pp. 1201-1204, 1998.

[25] C. Sanchez-Moreno, "Review: Methods used to evaluate the free radical scavenging activity in foods and biological systems," Food Sci. Tech. Int., vol. 8, pp. 121-137, 2002.

[26] C. Wentrup, "Reactive molecules," The Neutral Reactive Intermediates in Organic Chemistry, New York: John Wiley and Sons, 1984, ch. 2.

[27] F. Malki and A. Touati, "Study on antioxydant activity of Pyrimidinium Betaines," presented at the IUPAC World Chemistry Congress,Istanbul, Turkey, August 11-16, 2013.

[28] D. Ferreira, D. Slade, and J. P. J. Marais, "Flavans and Proanthocyanidins," in Flavonoids: Chemistry, Biochemistry and Applications, O. M. Anderson and K. R. Mark- ham, Eds. CRC Press, Boca Raton, 2006, pp. 553-616.

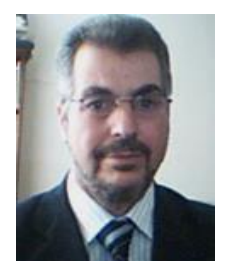

Saâd Moulay is an Algerian citizen, who received his bachelor's degree in chemistry from the Université des Sciences et Technologie Houari Boumèdiène USTHB (Algiers, Algeria) in 1979. He then received his Ph.D. degree in organic and polymer chemistry, under the supervision of Emeritus Professor William H. Daly, from the chemistry department of Louisiana State University (Baton Rouge, La, USA) in 1986. He is currently Professor of chemistry at Département de Chimie Industrielle of Université Saad Dahlab de Blida (Blida, Algeria).

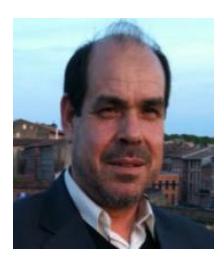

Abdelkader Touati was born in Algeria at Boumerdes on March 3, 1959. He received his bachelor's degree in chemistry from the Université des Sciences et Technologie Houari Boumèdiène USTHB (Algiers, Algeria) in 1984. He then received his Ph.D. degree in molecular organic chemistry from Paul Sabatier University (Toulouse, France) in 1988. He is currently Professor and researcher in chemistry at Département de Chimie of Ecole Normale Supérieure de Kouba, (Algiers, Algeria). 